\title{
Arbeit 4.0 in der Produktentstehung mit IviPep
}

\section{Identifizierung und ganzheitliche Umsetzung von Szenarien digitalisierter Arbeit}

\author{
Marc Foullois, Anna-Lena Kato-Beiderwieden $\mathbb{B}^{0}$, Lisa Mlekus $\mathbb{B}^{\circ}$ \\ Günter W. Maier $\mathbb{B}^{0}$, Sascha Jenderny, Carsten Röcker, Oliver Dietz, \\ Matthias Pretzlaff, Oliver Huxdorf, Friedrich von Dungern, \\ Dieter Bräutigam, Lars Seifert und Roman Dumitrescu
}

\subsection{Ausgangssituation und Zielsetzung}

Unternehmen des produzierenden Gewerbes werden zunehmend von Informationsund Kommunikationstechnik (IKT) durchdrungen [1]. Der Begriff Industrie 4.0 bringt diese vierte industrielle Revolution zum Ausdruck [2]. Historisch lässt sich beobachten, dass sich mit einer Veränderung der Wertschöpfung auch die Arbeitswelt verändert. Der

M. Foullois $(\bowtie) \cdot R$. Dumitrescu

Fraunhofer-Institut für Entwurfstechnik Mechatronik IEM, Paderborn, Deutschland

A.-L. Kato-Beiderwieden · L. Mlekus · G. W. Maier

Universität Bielefeld, Abteilung für Psychologie, Bielefeld, Deutschland

S. Jenderny $\cdot$ C. Röcker

Fraunhofer-Institut für Optronik, Systemtechnik und Bildauswertung IOSB,

Institutsteil für Industrielle Automation INA, Lemgo, Deutschland

O. Dietz

Diebold Nixdorf Systems GmbH, Paderborn, Deutschland

M. Pretzlaff

HELLA GmbH \& Co. KGaA, Operational Excellence \& Industrial Engineering,

Lippstadt, Deutschland

O. Huxdorf $\cdot$ F. von Dungern

INVENT GmbH, R\&D BUSINESS UNIT, Braunschweig, Deutschland 
derzeitige Wandel der Arbeitswelt, welcher durch die Adaption von digitalen Technologien geprägt ist, wird durch den Begriff Arbeit 4.0 beschrieben [3]. Technologien der Digitalisierung haben großes Potenzial, die Art und Weise, wie wir wirtschaften und arbeiten, grundlegend zu verändern [4]. Vor allem in der Produktentstehung verspricht die Digitalisierung ein hohes Nutzenpotenzial, da die neuartigen intelligenten technischen Systeme einen großen Zuwachs an Daten über den gesamten Produktlebenszyklus verfügbar machen. Grundsätzlich können vier Technologiefelder unterschieden werden: Erfassung, Verarbeitung und Analyse digitaler Daten; Automatisierung von Wertschöpfungsketten und Produkten; Vernetzung von Systemen und Virtualisierung [5]. Die Anwendung einer derartigen digitalen Technologie in der Arbeitswelt lässt sich in einem Anwendungsszenario digitalisierter Arbeit beschreiben. Remote Experten, digitaler Auftragsdurchlauf und Predictive Maintenance sind nur einige Beispiele, die zunehmend im produzierenden Gewerbe beobachtet werden können [5].

Die Einführung von Anwendungsszenarien digitalisierter Arbeit ist ein komplexes Handlungsfeld und geht mit tiefgreifenden Veränderungen in der Arbeitswelt einher. Unternehmensprozesse und -strukturen sowie Tätigkeiten und Kompetenzen der handelnden Personen müssen den neuen Anforderungen angepasst werden [6]. Dieses Bewusstsein, dass neben der technischen Perspektive auch die organisatorische und menschliche Perspektive elementare Stellhebel für die erfolgreiche Gestaltung einer digitalisierten Arbeitswelt sind, muss bei allen Beteiligten geschaffen werden. Die Berücksichtigung des soziotechnischen Spannungsfeldes aus Mensch-Organisation-Technik ist somit ein Erfolgsfaktor für die Einführung von Anwendungsszenarien digitalisierter Arbeit [7]. Aufgrund der Komplexität des Handlungsfeldes und der Vielzahl und Heterogenität der Anwendungsszenarien digitalisierter Arbeit fällt es Unternehmen schwer, die für sie geeigneten Anwendungsszenarien zu identifizieren. Zudem ist der konkrete Nutzen oftmals noch unklar. Aus diesem Grund wurde in dem Verbundprojekt „Instrumentarium zur Gestaltung individualisierter virtueller Produktentstehungsprozesse in der Industrie 4.0“ (IviPep) ein Instrumentarium zur humangerechten und wirksamen Gestaltung einer digitalisierten Arbeitswelt in der Produktentstehung erarbeitet. Dieses bildet einen Orientierungsrahmen für Unternehmen [8].

In Abb. 16.1 sind die Facetten des Forschungsvorhabens mit dem soziotechnischen Spannungsfeld aus Mensch-Organisation-Technik dargestellt. Durch die Forschungspartner Fraunhofer IEM, Fraunhofer IOSB-INA und Universität Bielefeld (Arbeits-

D. Bräutigam

HANNING ELEKTRO-WERKE, Oerlinghausen, Deutschland

L. Seifert

myview systems GmbH, Büren, Deutschland 


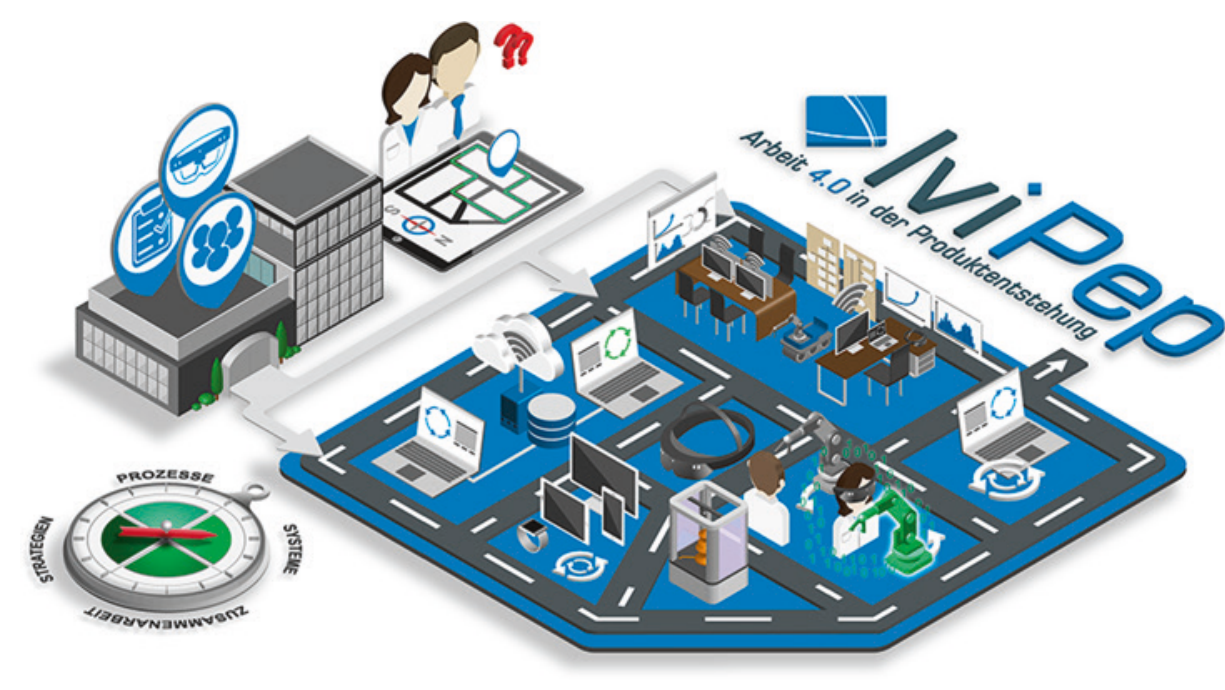

Abb. 16.1 Projektschaubild

und Organisationspsychologie) wurden unter anderem die Potenziale und Auswirkungen digitalisierter Arbeit analysiert. Hierzu wurden typische Herausforderungen in Unternehmen identifiziert und eine Bandbreite an Technologien auf ihren Nutzen hin analysiert. Die Auswirkungen der Einführung einer nutzenstiftenden Technologie auf die Prozesse, Tätigkeiten und Beschäftigten wurden daraufhin bewertet. Die Gestaltung digitalisierter Arbeit ist unternehmensindividuell. Ein strukturiertes Vorgehen zur Einführung der entsprechenden Anwendungsszenarien digitalisierter Arbeit unterstützt Unternehmen dabei, alle Einflussfaktoren der digitalisierten Arbeit zu berücksichtigen und somit die fortschreitenden Möglichkeiten der Digitalisierung zu nutzen. Das Einbeziehen der Beschäftigten in den Transformationsprozess ist hierbei von besonderer Bedeutung [9]. Die Technologieakzeptanz sowie die Qualifizierung und die Vorbereitung auf die Veränderungen in der Arbeitswelt sind zu berücksichtigen und wurden in dem Forschungsvorhaben analysiert und umgesetzt.

Die Einführung von Anwendungsszenarien digitalisierter Arbeit in den vier Pilotunternehmen Diebold Nixdorf Systems GmbH, HELLA KGaA Hueck \& Co., INVENT GmbH und HANNING ELEKTRO-WERKE GmbH \& Co. KG ermöglichten durch das Erproben der Konzepte und Befragungen der Beschäftigten einen facettenreichen und vielschichtigen Erkenntnisgewinn zum Wandel der Arbeitswelt. Die erarbeiteten Inhalte wurden in dem Instrumentarium festgehalten. Der Umsetzungspartner myview systems $\mathbf{G m b H}$ hat hierfür eine benutzerfreundliche Plattform erarbeitet, die interessierten Unternehmen die Möglichkeit bietet, von den Erkenntnissen aus dem Forschungsprojekt zu profitieren und diese auf den eigenen Produktentstehungsprozess (PEP) anzuwenden. 


\subsection{Forschungsergebnisse und Anwendungsbeispiele}

In diesem Kapitel werden sowohl Kernaspekte der Forschungsergebnisse sowie die Anwendungsbeispiele der Pilotunternehmen beschrieben. Hierzu wird zunächst der Prozess der Produktentstehung beschrieben, anhand dessen Szenarien digitalisierter Arbeit identifiziert werden können. Daraufhin wird auf die Charakteristika zur Beschreibung der Szenarien eingegangen. Erfolgsfaktor für die Einführung der Szenarien ist die Betrachtung des soziotechnischen Systems. Dieses beinhaltet neben der Perspektive der Technik auch die Perspektive Mensch und Organisation. Aus diesem Grund werden die Technologieakzeptanz und weitere Einstellungen der Beschäftigten sowie das Change-Management beschrieben. Damit die Beschäftigten entsprechend der neuen Anforderungen der digitalen Arbeitswelt vorbereitet sind, wird auf das Thema der Qualifizierung eingegangen. Abschließend zu den Forschungsergebnissen werden die vier Pilotunternehmen mit ihren Anwendungsbeispielen beschrieben.

Die Entwicklung von komplexen Marktleistungen kann nur durch ein Zusammenwirken verschiedener Fachdisziplinen (z. B. Marketing, Elektrotechnik, Maschinenbau, Softwaretechnik u. v. m.) erfolgen. Die Produktentstehung, von der Geschäftsidee bis hin zum Serienanlauf, benötigt somit einen Prozess, der das Zusammenwirken der Disziplinen gewährleistet. Der Prozess weist in der Realität in der Regel eine Reihe von Herausforderungen auf. Das Fehlen aktueller Konstruktionsstände für die Montageplanung ist ein Beispiel. Im Anschluss können die Herausforderungen hinsichtlich nutzenversprechender digitaler Technologien analysiert werden. Für das aufgeführte Beispiel ist eine nutzenversprechende Technologie Augmented Reality (AR), da diese die Möglichkeit besitzt, virtuelle Objekte in die reale Umgebung und somit in den realen Montagestationen zu platzieren.

Referenzprozess: Die Herausforderung in Kombination mit der digitalen Technologie bilden ein Anwendungsszenario digitalisierter Arbeit. In dem Projekt wurde der PEP der verschiedenen Unternehmen aufgenommen und hinsichtlich der Herausforderungen analysiert. Die aufgenommenen Prozesse wurden daraufhin durch einen abstrahierten Referenzprozess ersetzt, der ein Musterprozess für die Abläufe der Produktentstehung darstellt. Dieser Referenzprozess wurde entsprechend dem 4-Zyklen-Modell der Produkt- und Marktleistungsentstehung nach Gausemeier in die Hauptaufgabenbereiche der strategischen Produktplanung, Produktentwicklung, Dienstleistungsentwicklung und Produktionssystementwicklung gegliedert [10]. Die gesammelten Herausforderungen wurden mit nutzenversprechenden digitalen Technologien versehen und als Szenarien in dem Referenzprozess verortet. Zusammen bilden Referenzprozess und Szenarien somit eine Potenziallandkarte für die Digitalisierung der Arbeitswelt.

Referenzarchitektur: Zur Charakterisierung der Szenarien wurde eine Referenzarchitektur entwickelt. Diese besagt, dass ein Szenario digitalisierter Arbeit im Kern durch 5 Merkmale und die Zusammenhänge untereinander beschrieben wird. Im Mittel- 
punkt steht das Merkmal der Arbeitsaufgabe, welche es zu erledigen gilt. Dies kann beispielsweise eine manuelle Routineaufgabe sein. Die weiteren Merkmale sind die digitale Technologie (z. B. Kommunikationstechnologie), der Akteur (z. B. Mensch-System), die Herausforderung (z. B. Zeit) und die Situation (z. B. Tätigkeit) [5].

Technologieakzeptanz: Damit eine neue digitale Technologie von den Beschäftigten auch tatsächlich eingesetzt wird, ist die Technologieakzeptanz ein entscheidender Faktor. Das Technoloy Acceptance Model (TAM) [11] umfasst Faktoren wie interindividuelle Benutzermerkmale und den beruflichen Kontext (z. B. Freiwilligkeit), die die Nutzung von Informationstechnologien vorhersagen. Auf die Technologieakzeptanz haben neben diesen Faktoren aber auch Merkmale der Technologie einen Einfluss. Daher wurde im Projekt das TAM um Eigenschaften der User-Experience erweitert, damit auch die technologiebezogenen Merkmale miterfasst werden [12]. Die Merkmale Output-Qualität, Durchschaubarkeit, Zuverlässigkeit und Neuartigkeit zeigten sich als zusätzliche signifikante Prädiktoren für die Technologieakzeptanz. Das Modell kann von Technologieentwicklern, Change-Managern und den Akteuren selbst eingesetzt werden.

Einstellungen der Beschäftigten: Der Einsatz einer neuen digitalen Technologie kann Einfluss auf die Einstellungen der Beschäftigten haben. Ebenso können auch die Einstellungen der Beschäftigten beeinflussen, wie gut die Einführung einer neuen Technologie gelingt. In den beiden Pilotunternehmen HELLA und INVENT wurden vor Einführung der neuen Technologie u. a. die Technologieaffinität und -ängstlichkeit erhoben, da diese gerade am Anfang Einfluss darauf nehmen, wie eine neue Technologie wahrgenommen wird und somit wie hoch die Technologieakzeptanz ist. Die Ergebnisse der Befragungen zeigten, dass drei viertel der Beschäftigten positive Einstellungen zu neuen Technologien hatten, welche eine gute Grundlage für die Einführung einer neuen Technologie bildeten. Durch eine Befragung bei HELLA konnte gezeigt werden, dass bestimmte Arbeitsgestaltungsmerkmale mit positiven Arbeitseinstellungen zusammenhängen. Dort zeigte sich, dass in einem Mixed-Mock-Up-Workshop insbesondere Aufgabenmerkmale wie Autonomie und Ganzheitlichkeit sowie die soziale Unterstützung mit positiven Arbeitseinstellungen (Arbeitszufriedenheit, intrinsische Arbeitsmotivation, verringertes Stresserleben) einhergingen [13]. Die Einführung einer neuen Technologie hat allerdings nicht unter allen Umständen Auswirkungen auf die Arbeitseinstellungen, wie sich in einer Befragung in einem Unternehmen (INVENT) herausstellte. Durch eine Befragung vor und nach der Einführung einer neuen Technologie wurde deutlich, dass sich die Arbeitseinstellungen der Beschäftigten durch die Einführung der Technologie nicht bedeutsam verändert haben. Ein möglicher Einflussfaktor könnte sein, wie stark die Technologie die Arbeit verändert und wie viel Zeit bei der Arbeit mit der Technologie gearbeitet wird.

Change-Management: Mit der Einführung einer neuen digitalen Technologie in der Arbeitswelt gehen Veränderungen der Strukturen, Prozesse oder Verhaltensweisen in einer Organisation einher. Die Maßnahmen zu den Veränderungen sowie die Verfolgung 
der Änderungen werden unter Change-Management zusammengefasst. Ein zentrales Ziel von Change-Management im Rahmen einer Technologie- oder Prozesseinführung ist die Akzeptanz und somit die nachhaltige Implementierung der Technologie bzw. des Prozesses. Um dies zu gewährleisten, ist es wichtig, die zukünftigen Akteure in einem frühen Stadium der Einführung einzubinden [14]. Bei HELLA erfolgte dies in Form eines Workshops. Die Beschäftigten, die in Zukunft mit dem Mixed Mock-Up arbeiten sollten, konnten in dem Workshop den aktuellen Entwicklungsstand der Technologie ausprobieren und Änderungswünsche in Bezug auf die Gestaltung sowie mögliche Einschränkungen in Arbeitsabläufen aufgrund der Technologie äußern. Die Möglichkeit der Partizipation hat zu einer Steigerung der Veränderungsbereitschaft bei den Workshopteilnehmer*innen geführt [13]. Eine weitere Change-Management-Aktivität erfolgte bei HANNING. Bei der dortigen Schulung zur Umstellung des Konformitätsbewertungsprozesses wurde den Beschäftigten neben der inhaltlichen Schulung die Möglichkeit gegeben, sich zu dem neuen Prozess zu äußern. Konkret wurden hier die Befürchtungen in Bezug auf den neuen Prozess (z. B. höherer Zeitaufwand) sowie mögliche Vorteile gegenüber dem alten Prozess (z. B. geringere Angreifbarkeit) gesammelt. Abschließend wurden Lösungsstrategien erarbeitet, damit die erwarteten Befürchtungen nicht eintreten.

Im Weiteren wurden im Rahmen des Projektes Interviews mit ausgewählten Experten der Pilotunternehmen zum Thema Veränderungskultur geführt. Die Untersuchungen zielten neben einer rein quantitativen Abfrage von Erfolgsfaktoren insbesondere auf eine Momentaufnahme aktueller Anforderungen und Herausforderungen in Veränderungsprozessen ab. Mithilfe der Interviews konnten sowohl Erfolgs- als auch Misserfolgsfaktoren für Veränderungsprozesse identifiziert werden. Hierbei bestätigen die Forschungsergebnisse die These, dass Change-Management als Gesamtprozess gelebt werden muss und eine (technologische) Veränderung sowohl von Beschäftigten angenommen als auch vom Management vorgelebt werden muss, um langfristig erfolgreich zu sein. Interessant ist jedoch, dass die Strategien der Interviewpartner jeweils individuell für das eigene Unternehmen sind. Wenngleich auf Heuristiken und Erfahrungen (d.h. bestehende Forschungsergebnisse) zurückgegriffen werden kann, wird die Notwendigkeit kontext- und situationsbezogener Ansätze der Führung und der Organisation im Ganzen, insbesondere vor dem Hintergrund sich immer schneller entwickelnder Technologien, immer deutlicher.

Qualifizierungsmaßnahmen: Aufgrund bisheriger Studien [15] und theoretischer Überlegungen zum soziotechnischen System ist davon auszugehen, dass sich die Einführung einer neuen Technologie darauf auswirkt, welche Fähigkeiten, Fertigkeiten und Kenntnisse die Beschäftigten für ihre Arbeit benötigen. In der Folge sind Qualifizierungsmaßnahmen notwendig. Um diese Veränderungen zu ermitteln, wurden in den Pilotunternehmen über Interviews und Fragebögen Anforderungsanalysen nach dem Vorgehen der Task Analysis Tools [16] durchgeführt. Ist die geplante Technologie noch nicht eingeführt, gibt es auch die Möglichkeit eine Variante dieser Methode für einen zukünftigen Arbeitsplatz durchzuführen [17]. Die Ergebnisse zeigen, dass die 
Arbeit mit einer Technologie neben fachspezifischen Kenntnissen (z. B. zu Produkten und Materialien) in allen untersuchten Unternehmen und Abteilungen eine gewissenhafte Arbeitsweise erfordert. Gewissenhaftigkeit war jeweils unter den fünf bedeutsamsten Anforderungen. Beispielhaft ist in Abb. 16.2 das Anforderungsprofil eines Projektpartners abgebildet. Nach Einführung einer neuen Technologie zeigten sich insbesondere bei den Anforderungen „Fachkompetenz Material- und Bauteilprüfung”, „Planungsfähigkeit” und ,räumliches Vorstellungsvermögen” große Veränderungen im Vergleich zu der vorherigen, analogen Arbeit.

Infolge der veränderten Anforderungen an die Beschäftigten, wurden in den Pilotunternehmen Schulungen durchgeführt. Ziel war es, die Beschäftigten einerseits im Umgang mit den neuen Technologien zu schulen und andererseits veränderte Anforderungen im PEP im Allgemeinen zu meistern. Themen waren der Einsatz und die Anwendung von AR-Technologien, die Vermittlung eines neuen Konformitätsbewertungsprozesses, der Einsatz von Kreativitätstechniken in der Produktentwicklung, die bedarfsgerechte Darstellung von Informationen in der kollaborativen Arbeit und die Bedienung von AR-Brille und Datenhandschuh im Mixed Mock-Up. Der Fokus in den Schulungen lag auf praktischen Übungseinheiten, um die Inhalte zu veranschaulichen und den Transfer des Geübten auf die berufliche Praxis zu gewährleisten. Bei der Schulung zum Einsatz von Kreativitätstechniken wurde eine Befragung mit den zehn Teilnehmenden durchgeführt. Es zeigte sich, dass diese bisher vornehmlich mit

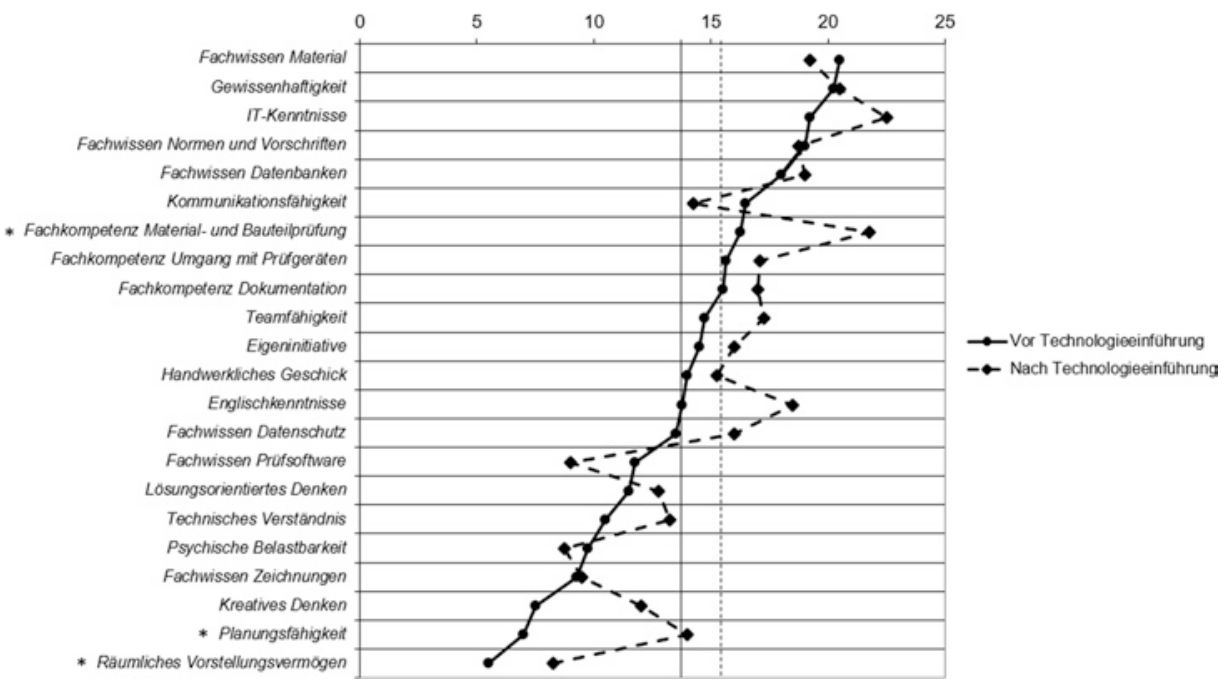

Abb. 16.2 Anforderungsprofil eines Projektpartners. Erhebung mit vier Personen vor und nach der Technologieeinführung. Vertikale durchgezogene Linie zeigt den Mittelwert vor Technologieeinführung an, vertikale gestrichelte Linie zeigt den Mittelwert nach Technologieeinführung an. * kennzeichnet Anforderungen, bei denen es vor und nach der Technologieeinführung große Veränderungen gab 
Techniken gearbeitet haben, die auf der Methode der freien Assoziation basieren. Von den zehn in der Schulung vermittelten Techniken war entsprechend ein Großteil nur zwei bis drei Personen bekannt, sodass hier von einer Erweiterung des methodischen Repertoires der Teilnehmenden auszugehen ist (für weitere Informationen siehe https:// www.ivipep.de/elementor-384/).

Nachdem mit dem Referenzprozess und der Referenzarchitektur, der Nutzerakzeptanz, dem Change-Management und der Qualifizierungsmaßnahmen Kernelemente für die Einführung digitalisierter Arbeit beschrieben wurden, werden im Folgenden die vier Anwendungsfälle der Pilotunternehmen beschrieben.

Diebold Nixdorf Systems GmbH - Einsatz innovativer und intelligenter Entwicklungsansätze innerhalb komplexer PEPs von intelligenten technischen Systemen Diebold Nixdorf ist weltweit führender Anbieter von IT-Lösungen und -Services für Retailbanken und Handelsunternehmen. Zu Beginn des Projektes IviPep wurden die Anforderungen an den digitalen Prototyp „NextGen-ATM“ in einem Konzeptentwurf konkretisiert. Als Ergebnis des Konzeptentwurfes wurde eine modellbasierte Beschreibung festgelegt, wobei das Anforderungs- und Projektmanagement in Konformität des internen PEPs in einer gesonderten Projektmanagementsoftware erfasst wurde. Die Datenzusammenführung erfolgte in einer Product-Lifecycle-Management (PLM) -Instanz. Hier wurden die modellbasierten Daten und Anforderungen mit den klassischen Entwicklungsdaten (CAD, CAE, CAM, VR) zusammengeführt. Aus den so zusammengeführten Informationen entstand zur ganzheitlichen gemeinsamen Produktbetrachtung eine auf AR-Technologie basierende Visualisierung. Das Vorhaben wurde am „NextGenATM" erfolgreich umgesetzt. Dieser wurde parallel in Form eines physikalischen Demonstrators mit der Bezeichnung Cash Cube realisiert. Dieser Demonstrator erhielt von der ATM Industry Association (ATMIA) den Global Innovation Award (1. Platz).

Das Ergebnis ist somit ein erprobtes digitales Hilfsmittel, welches zur Unterstützung der Beschäftigten in frühen Entwicklungsphasen intelligente technische Systeme visualisiert und die Projektbeteiligten bei Entscheidungsfindungen unterstützt. Besonderes Augenmerk galt im Forschungsvorhaben neben dem Zusammenführen und Bereitstellen von Informationen dem Visualisieren von entwicklungsrelevanten Daten mithilfe der AR-Technologie. Dies wurde im Projektverlauf als sehr positiv durch die Mitarbeitenden bewertet. Begleitende arbeitspsychologische Untersuchungen bestätigen dies zum Abschluss des Projektes nachhaltig. Herausforderungen werden bei der effizienten Bereitstellung der CAD-Daten zur Visualisierung mit AR-Technologie aufgrund der hohen zu konvertierenden Datenmengen gesehen. Steigerungen der Leistungsfähigkeit zukünftiger AR-Hardware wird dies mitunter kompensieren.

Das Verwenden von AR-Technologie hat sich bei Diebold Nixdorf durch das Forschungsvorhaben IviPep in Bereichen der Produktentwicklung und des Produktmanagements etabliert. Die Designabteilung und die Vorentwicklung verwenden ARTechnologie zur Bereitstellung hochwertiger visueller Information; verschiedene am Entwicklungsprozess beteiligte Bereiche nutzen die Grundlage zur gemeinsamen Ent- 
scheidungsfindung im Projektverlauf. Daneben ist durch das Projekt die AR-Technologie bei Messeauftritten der Diebold Nixdorf zum festen Bestandteil avanciert. Die Kommunikation zum Kunden konnte erfolgreich optimiert werden und auch das Ausstellen der Exponate am Messeplatz konnte so in der Anzahl verringert werden. Dies spart Transporte und trägt somit aktiv zum Umweltschutz bei.

\section{HELLA KGaA Hueck \& Co. - Mixed Mock-Up zur Produktionssystemplanung}

HELLA ist international operierender Automobilzulieferer und auf innovative Lichtsysteme und Fahrzeugelektronik spezialisiert. In der klassischen Planung von Produktionssystemen wird ein Montagearbeitsplatz in einem sogenannten Mock-Up aus Kartonage nachgestellt. Die Verwendung von Hardware mit AR-Technologie soll die klassische Planung zukünftig in Form einer Mixed Mock-Up Anwendung um virtuelle Elemente erweitern. Der Mixed Mock-Up-Demonstrator ist ein Prototyp, der im Unternehmen HELLA eingesetzt werden soll.

In der ersten Projektphase wurde ein Demonstrator entwickelt, der das grundlegende Potenzial eines Mixed Mock-Ups aufzeigen sollte. In einer fest vorgegeben Sequenz konnten hier einige Bauteile zu einer Baugruppe gefügt werden und wurden so einem breiten Publikum u. a. Hella-intern und auf der Hannover Messe zugänglich gemacht. Dieser Demonstrator wurde in der zweiten Projektphase zu einem Funktionsprototyp erweitert. Mit diesem ist es möglich, die Bauteile in einer beliebigen Reihenfolge zu montieren. Um die Bauteile aus der CAD-Geometrie in das AR-Format zu überführen, wurde mithilfe einer Standard-Software der Konvertierungsprozess beschrieben und bei Hella on-premise umgesetzt. Die Bauteile lassen sich in der Anwendung in einem Auswahlmenü in die AR-Umgebung laden. Im Konfigurationsmodus können die konvertierten Bauteile nach ergonomischen Aspekten um den Arbeitsplatz angeordnet werden und diese Anordnung kann gespeichert werden. Im Montagemodus kann die Montage erprobt werden. Änderungswünsche in der Materialkonfiguration können dann wieder im Konfigurationsmodus verändert und erneut gespeichert werden. Über das MTM-UAS-Modul (Methods-Time Measurement - Universelles Analysier-System) können Veränderungen in der Materialanordnung nach MTM bemessen werden. Das heißt, es werden Zeiten für die Abläufe erfasst und optimiert. Eine optimale Konfiguration kann per Screenshot oder Videoaufzeichnung für weitere Zwecke dokumentiert werden.

Erste Tests anhand eines realen Produktprojekts ergaben durchaus positive Erkenntnisse für den Projektverlauf. So waren die Akteure von den Möglichkeiten und dem Potenzial der Anwendung sehr beeindruckt. Da sich die Anwendung noch im Entwicklungsstadium befindet, hindern die Usability und das Zusammenspiel der verschiedenen Hardwarekomponenten aktuell noch eine intuitive und pragmatische Nutzung der Anwendung.

Die neue Hardwaregeneration der AR-Brille setzt in Sachen Usability und Bedienkomfort einen neuen Meilenstein. Mit der Überführung der Module des Funktionsproto- 
typs auf die neue Hardware wird die Anwendung auf ein Level gebracht, welches die spontane und intuitive Nutzung der Anwendung erlaubt.

\section{INVENT GmbH - Digitales Werkzeug für die Entwicklung von Satellitenstrukturen}

Als anerkannter Leichtbau-Spezialist für innovative Faserverbundtechnologien der Branchen Luft- und Raumfahrt, Maschinenbau, Automotive, Schienenfahrzeuge und Schiffbau entwickelt und produziert die INVENT GmbH hochpräzise Strukturkomponenten, von der ersten Idee bis zur Serienfertigung. Dabei ist eine enge Verzahnung aller Beschäftigten und Disziplinen notwendig. Die Digitalisierung der administrativen wie auch fertigungstechnischen Unternehmensprozesse ist dabei ein wesentlicher Baustein um die zukünftige Verzahnung weiter $\mathrm{zu}$ intensivieren und die Wettbewerbsfähigkeit zu erhöhen.

Im Rahmen des Projektes wurden die bestehenden Strukturen des Unternehmens analysiert. Aus diesem Prozess wurden zwei wesentliche Schlüsseltechnologien zur weiteren Digitalisierung des Unternehmens identifiziert. Zum einen wurde parallel zum bestehenden Datenbanksystem des Unternehmens ein umfangreicheres, vielversprechendes und individualisierbares dokumentenbasiertes Datenbanksystem namens Limbas eingeführt. Dieses System wurde mit einem begrenzten Beschäftigtenkreis erprobt. Es ermöglicht eine engere Vernetzung aller organisatorischen Unternehmensprozesse. Vorausgesetzt weiterer positiver Ergebnisse soll dieses System im Anschluss an das Projekt weiter detailliert, erprobt und schlussendlich für alle Beschäftigten freigeschaltet werden.

Zum anderen wurden Facesheets für raumfahrttypische Sandwichpanele angefertigt. Hierfür wurden abweichend zum bisherigen Prozess ausschließlich digitale Fertigungsdokumente verwendet. Durch die Berücksichtigung der raumfahrttypischen Anforderungen und Prozesse konnte ein großer Querschnitt der Fertigung abgedeckt und deren Beeinflussung untersucht werden. Hierbei konnten viele positive aber auch einige negative Aspekte identifiziert werden, sodass eine weitere Erprobung im Nachgang des Projektes erforderlich ist.

Nach Abschluss des Projektes strebt die INVENT GmbH die Überarbeitung der eigenen Prozesse durch den zweckmäßigen Einsatz der erarbeiteten Technologien an. Dadurch werden der Know-How-Vorsprung gesichert und die Wettbewerbsfähigkeit erhöht.

\section{HANNING ELEKTRO-WERKE GmbH \& Co. KG - Digitale Technologie zur Unterstützung des Konformitätsmanagements}

HANNING ist auf kundenspezifische Antriebssysteme und -komponenten weltweit spezialisiert. Das Material Compliance Management und die Erstellung von CE-Konformitätserklärungen erfolgte bei HANNING bisher mithilfe umfangreicher Excel-Tabellen, die redundante Daten beinhalteten und nicht mehr handhabbar waren. Um die Prozesse zu vereinfachen, wurde mit der Datenbanktechnik ,semantisches Netz” ein Tool eingeführt, das bei der Analyse von Materialeigenschaften unterstützt. 
Durch eigenfinanzierte Beauftragung eines Software-Herstellers wurden die Programmierung des semantischen Netzes und die Gestaltung der Masken-Oberflächen beauftragt. Um möglichst anwendungsfreundliche Dashboards und Abfrage-Menüs zu erzielen, wurden die in der Aufgabe Material Compliance und CE-Konformität zuständigen Beschäftigten eng in die Entwicklung eingebunden. Prototypen wurden mit realen HANNING-Daten geladen und Alltagsaufgaben darin abgearbeitet. Auswirkungen dieses digitalisierten Arbeitens wurden durch Arbeitspsychologinnen der Universität Bielefeld bei den involvierten Beschäftigten erfragt und ausgewertet.

Die Nutzung der Datenbank-Technik „semantisches Netz“ als Tool in einer digitalisierten Arbeitswelt bewerten wir als sehr positiv. Die Zeitersparnis bei der Analyse, ob ein HANNING-Produkt die Materialeigenschaften bzgl. der Abfrage nach verbotenen Stoffen und geächteten Substanzen erfüllt, ist deutlich. Zugleich werden Stressmomente abgebaut, da die Verlässlichkeit der richtigen Analyseergebnisse datenbank-technisch gewährleistet wird. Denn sowohl die Material Compliance als auch die CE-Konformitätserklärung werden mit rechtsverbindlichen Unterschriften ausgestattet als Deklarationsdokumente an den Kunden gegeben. Insofern besteht eine hohe Qualitätsanforderung an die Richtigkeit der Analyse, um Schadensersatzforderungen durch Kunden ausschließen zu können.

Die operative Nutzung der im Kontext IviPep gestarteten Graphdatenbank ist für HANNING beschlossene Sache. Der Ausbau dieses semantischen Netzes in viele Richtungen ist möglich (z. B. durch Dashboards) und eine Frage der jährlichen Budgetplanung bei HANNING.

Instrumentarium: Die Erkenntnisse aus der Einführung von Anwendungsszenarien digitalisierter Arbeit in den vier Pilotunternehmen, wurden zusammen mit den erarbeiteten Konzepten und weiteren identifizierten Anwendungsszenarien aus Forschung und Praxis in dem Instrumentarium gebündelt (https://ivipep.myview.de). Das Instrumentarium stellt eines der Kernergebnisse des Forschungsvorhabens dar und wurde von den Forschungs- und Industriepartnern in Zusammenarbeit mit dem Umsetzungspartner myview systems entwickelt. Abb. 16.3 zeigt die Landingpage des Instrumentariums. Die einzelnen Aspekte und Funktionen werden nachfolgend erläutert.

Kernelement des Instrumentariums ist eine Datenbank aus identifizierten, beschriebenen und bewerteten Anwendungsszenarien digitalisierter Arbeit. Auf diese kann über die Direktsuche direkt zugegriffen werden. Diese bietet einen strukturierten Zugang zu allen Steckbriefen der Anwendungsszenarien. Zusätzlich können die Szenarien über eine Freitextsuche oder über Kriterien (Verortung im PEP, Investitionen und Technologie) gefiltert werden.

Die Anwendungsszenarien wurden über verschiedene Ansätze identifiziert. Zum einen wurde eine umfassende Literaturrecherche durchgeführt, wodurch bereits existierende sowie konzeptionell beschriebene Anwendungen digitaler Technologien in der Arbeitswelt der Produktentstehung und angrenzender Bereiche zusammengetragen wurden. Zum anderen wurden Workshops mit Industrievertretern durchgeführt, bei denen 

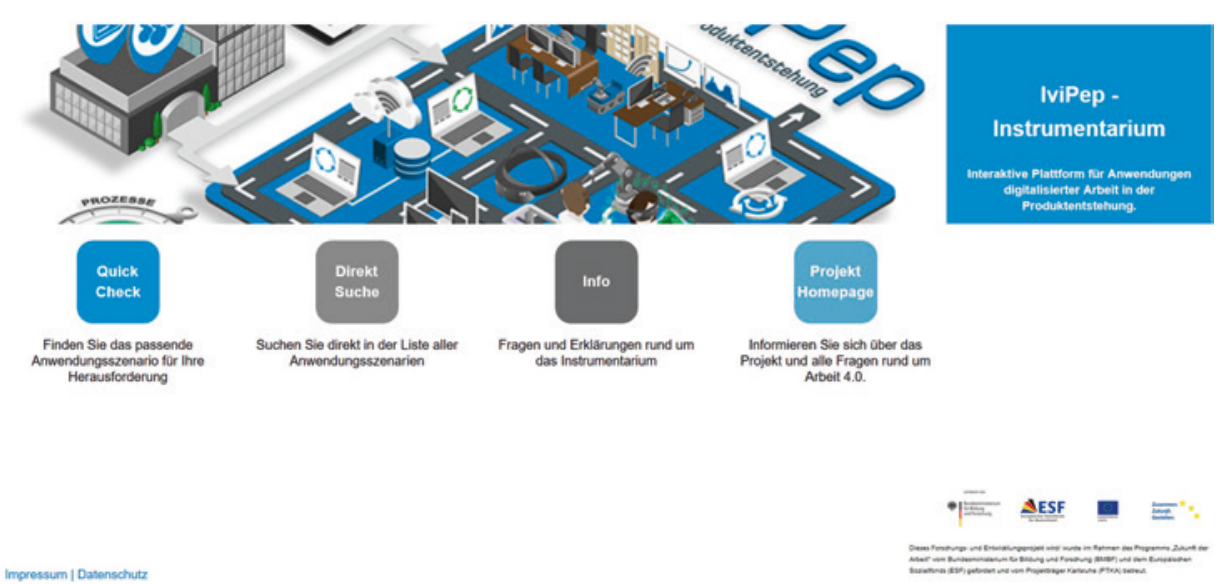

Abb. 16.3 IviPep-Instrumentarium Landingpage

Herausforderungen in der Produktentstehung identifiziert und die Anwendung nutzenversprechender Technologien diskutiert wurden. Im Anschluss an die Identifikation wurden die vorhandenen Anwendungsszenarien beschrieben. Hierzu wurde eine grundlegende Beschreibung des Anwendungsfalls sowie des Ablaufs des Szenarios vorgenommen. Zusätzlich wurden die Anwendungsszenarien durch weitere Informationen ergänzt, (z. B. Technologie, Prozessschritt). Abschließend wurden die Anwendungsszenarien anhand eines Kriterienkatalogs aus soziotechnischen Kriterien, welche die Dimensionen Mensch, Organisation und Technik umfassen, bewertet. Hierzu wurden insgesamt 25 Kriterien von Expert*innen identifiziert, welche sich in mehrere Faktoren unterteilen. Weitere Ausführungen zu dem Kriterienkatalog können in Jenderny et al. und Mlekus et al. nachgelesen werden [18, 19]. Die Abb. 16.4 zeigt die Darstellung eines exemplarischen Anwendungsszenarios digitalisierter Arbeit in dem Instrumentarium.

Ein weiteres Kernelement des Instrumentariums ist der Quick Check. Dieser ermöglicht es Unternehmen, spezifische Anwendungsszenarien für ausgewählte Herausforderungen zu identifizieren. Hierbei kann aus acht Herausforderungen ausgewählt und in Verbindung mit der Verortung im PEP gefiltert werden. Im Hintergrund wird dann ein Match mit den Anwendungsszenarien vorgenommen. Die vorgeschlagenen Anwendungsszenarien haben das Potenzial, die Herausforderung zu lösen oder die Beschäftigten bei der Lösung zu unterstützen. Unternehmen werden so in die Lage versetzt, schnell und effektiv für sie passende Lösungen für ihre Herausforderungen zu finden. Weitere Aspekte des Instrumentariums sind eine Weiterleitung zur Projektwebsite (www.ivipep.de) sowie eine Infoseite, die weiterführende Erläuterungen zum Instrumentarium beinhaltet.

Die myview systems als Umsetzungspartner hatte das Ziel, das Instrumentarium über eine Online-Plattform abzubilden, umzusetzen und im Projekt einzuführen. Mit 


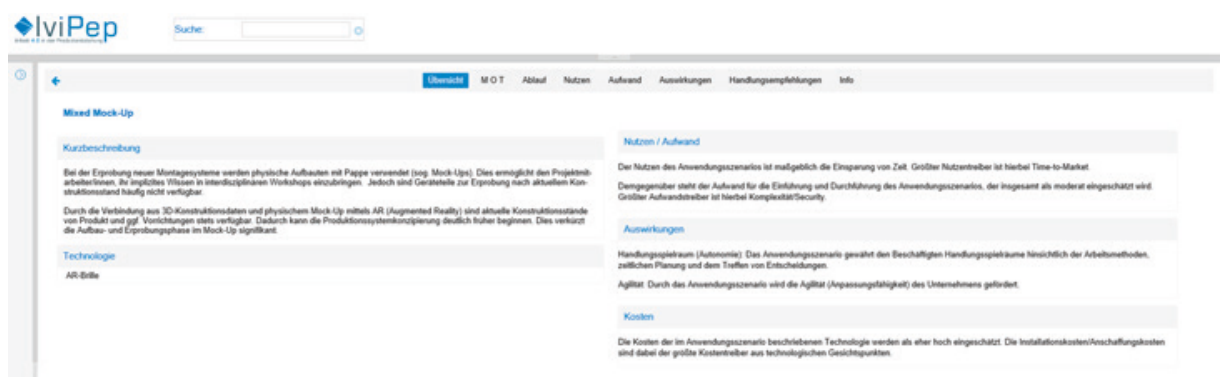

Abb. 16.4 Darstellung eines exemplarischen Anwendungsszenarios digitalisierter Arbeit in dem Instrumentarium

über 15 Jahren Erfahrung im Bereich der datenbankgestützten Produktkommunikation zählt myview systems zu den etablierten Anbietern von Produkten und Lösungen zum Katalog- und Produktinformationsmanagement. Die Kompetenzen von myview systems passen somit zu den Anforderungen an die Entwicklung eines Instrumentariums mit einem intuitiven Front-End mit einer Wissensdatenbank als Back-End. Nach der Anforderungsaufnahme an die Plattform wurde das Konzept mit der Architektur des Instrumentariums entwickelt.

Die Architektur des Instrumentariums besteht zum einen aus einer Workbench. Diese dient als Erfassungs- und Modellierungswerkzeug. In der Workbench werden die Anwendungsszenarien zusammen mit den zugehörigen Metadaten als einzelne Datensätze angelegt. Hierzu wurde eine Importschnittstelle über Excel eingerichtet. Die Workbench selbst ist aus den Modulen myview xmedia DataManager und myview xmedia ViewEditor aufgebaut und diente in den frühen Phasen des Projektes als Abstimmungswerkzeug mit dem Konsortium. Eine weitere Kernkomponente der Architektur ist der Store. Diese Datenablage stellt das Back-End bereit. Sie besteht aus einer innovativen „Backend as a Service“ Infrastruktur. Store und Workbench sind über einen XML DatenSynchronisationsmechanismus verbunden. Als dritte Grundkomponente wurde eine App auf der Basis dynamischer HTML-Technologie implementiert. Diese greift die Daten aus dem Store über eine Rest API ab. Die App ermöglicht die Bereitstellung einer intuitiv bedienbaren Web-Applikation. Die Einführung in den Pilotunternehmen erfolgte über Veranstaltungen wie Begleitkreistreffen.

\subsection{Ausblick}

Wenngleich die Arbeit im Projekt IviPep die zuvor gestellten Forschungsfragen in Hinblick auf die Interdependenzen zwischen Mensch, Technik und Organisation im PEP behandelt hat, ergeben sich aus der Arbeit weitere Forschungsfelder, die es zu erschließen gilt. Beispielsweise gilt es festzustellen, ob sich in einem Bereich, der 
nicht PEP ist, ähnliche oder andere Forschungsergebnisse vermuten lassen. Denkbar sind andere Bereiche der (industriellen) Arbeit, wie die Fertigung oder die berufliche Aus- und Weiterbildung, da hier ähnliche Technologien genutzt werden und oftmals ähnliche Tätigkeiten durchgeführt werden müssen, die (menschlichen und technologischen) Anforderungen jedoch von denen eines klassischen PEPs abweichen können. Eine weitere Forschungsfrage ergibt sich aus der Feststellung, dass die im Projekt eingeführten Technologien und Werkzeuge in den jeweiligen Anwendungsfeldern einen vergleichsweise geringen Teil des gesamten Arbeitsprozesses ausgemacht haben. Hierbei ist zu prüfen, ob eine höhere Präsenz der Technologie im Arbeitsprozess gleichbedeutend mit stärker ausgeprägten Veränderungen des Arbeitsprozesses für Beschäftigte ist.

Mögliche Synergien können des Weiteren zu bestehenden Vorhaben im Bereich der künstlichen Intelligenz geschaffen werden. Hierbei sind insbesondere die Leitthemen der autonomen Generierung von Maschinendaten sowie der optimierten Entscheidungsfindung für menschliche Akteure durch die Visualisierung dieser Daten interessant. Hierbei können, neben der entwickelten Softwarelösung, auch die im Projekt IviPep erarbeiteten methodischen Kenntnisse, wie etwa die Workshops zur Erfassung neuer Szenarien [20], einen wertschöpfenden Beitrag leisten.

Ebenfalls kann der im Projekt verfolgte partizipative Ansatz für die Arbeit in bestehenden und kommenden Projekten genutzt werden. So beschäftigt sich das derzeit laufende Projekt it's OWL-AWARE mit unternehmensübergreifenden Lernplattformen und Wissensdistribution im Kontext der Personalentwicklung sowie der Anwendung partizipativer Methoden im Kontext innovativer Technologieentwicklung. Auch hier ergeben sich Ansatzpunkte zur Weiterentwicklung der im Projekt IviPep verorteten Forschungsfragen. So ist beispielsweise eine Weiterentwicklung des Instrumentariums von einer passiven Informationsstelle zu einer Plattform möglich, welche die aktive Teilhabe des Mittelstandes am digitalen Wandel fördern und die „Leuchtturmwirkung“ des Industrie- und Innovationsstandortes Ostwestfalen-Lippe stärken kann. Hiermit verbunden kann insbesondere dem in der Region ansässigen Mittelstand eine Möglichkeit des Erfahrungsaustausches gegeben und der Technologietransfer gefördert werden. Im Hinblick auf eine solche Art der digitalen Teilhabe können sich weitere Forschungsarbeiten ferner insbesondere an bestehenden Modellfabriken und Reallaboren (SmartFactoryOWL, Lemgo Digita. SE Live Lab) orientieren. Auch die im Projekt entwickelten Schulungsund Weiterbildungsformate können zur nachhaltigen Kompetenzförderung über bereits bestehende (vgl. Transferprojekte des Spitzenclusters it's OWL) oder zu etablierende Transferinstrumente überführt werden.

\subsection{Weiterführende Projektinformationen}

Informationen zu dem Projekt, den Projektpartnern, Veröffentlichungen sowie Veranstaltungen können auf der Projekthomepage gefunden werden (www.ivipep.de). 
Das Instrumentarium mit den Anwendungsszenarien digitalisierter Arbeit aus Forschung und Praxis kann über einen Webzugang aufgerufen werden (https://ivipep. myview.de).

\section{Projektpartner und Aufgaben}

- Fraunhofer-Institut für Entwurfstechnik Mechatronik IEM Digitalisierter Produktentstehungsprozess auf Basis von Szenarien digitalisierter Arbeit

- Fraunhofer-Institut für Optronik, Systemtechnik und Bildauswertung IOSB, Institutsteil für industrielle Automation INA

Mensch-Technik-Interaktion innerhalb von Szenarien digitalisierter Arbeit

- Universität Bielefeld, Arbeits- und Organisationspsychologie

Konzept zur humanzentrierten Gestaltung digitaler Produktentstehung

- Diebold Nixdorf Systems GmbH

Einsatz innovativer und intelligenter Entwicklungsansätze innerhalb komplexer Produktentstehungsprozessen von intelligenten technischen Systemen

- HELLA KGaA Hueck \& Co.

Mixed Mock-Up zur Produktionssystemplanung

- INVENT GmbH

Digitales Werkzeug für die Entwicklung von Satellitenstrukturen

- HANNING ELEKTRO-WERKE GmbH \& Co. KG

Digitale Technologie zur Unterstützung des Konformitätsmanagements

- myview systems GmbH

Vernetzte Wissensplattform für das IviPep-Instrumentarium

\section{Literatur}

1. Bundesministerium für Wirtschaft und Energie (Hrsg) (2014) Monitoring-Report Digitale Wirtschaft 2014. Innovationstreiber IKT. https:/www.bmwi.de/Redaktion/DE/Publikationen/ Digitale-Welt/monitoring-report-digitale-wirtschaft-2014.html. Zugegriffen: 31. März 2020

2. Bundesministerium für Wirtschaft und Energie (Hrsg) Was ist Industrie 4.0? https://www. plattform-i40.de/PI40/Navigation/DE/Industrie40/WasIndustrie40/was-ist-industrie-40.html. Zugegriffen: 31. März 2020

3. Brynjolfsson E, McAfee A (2014) The second machine age: Work, progress, and prosperity in a time of brilliant technologies. WW Norton \& Co, New York

4. Altemeier K, Bansmann M, Dietrich O, Dumitrescu R, Nettelstroth W (2017) Auf dem Weg zu Industrie 4.0: Gestaltung digitalisierter Arbeitswelten. https://www.its-owl.de/fileadmin/PDF/ Publikationen/2017_Broschuere_Arbeit40.pdf. Zugegriffen: 31. März 2020

5. Berger R (Hrsg) Die Digitale Transformation der Industrie. https://bdi.eu/media/user_upload/ Digitale_Transformation.pdf. Zugegriffen: 31. März 2020

6. Bansmann M, Foullois M, Wöste L, Bentler D, Paruzel A, Mlekus L, Jenderny S, Dumitrescu R, Maier GW (2019) Arbeitsplatzplanung mit Augmented Reality und ein Dienstleistungs- 
system im Konformitätsmanagement als Anwendungsszenarien in der industriellen Praxis. In: Bosse CK, Zink KJ (Hrsg), Arbeit 4.0 im Mittelstand. Chancen und Herausforderungen des digitalen Wandels für KMU. Springer Gabler, Wiesbaden, S 197-217

7. Porter ME, Heppelmann JE (2015) How smart, connected products are transforming companies. Harvard Bus Rev 93(10):96-114

8. Ulich E (2011). Arbeitspsychologie. vdf Hochschulverlag, Zürich

9. Kato-Beiderwieden A-L, Mlekus L, Foullois M, Jenderny S, Röcker C, Maier GW (2020) Instrumentarium zur Gestaltung digitalisierter Arbeit. In: Gesellschaft für Arbeitswissenschaft e.V. (Hrsg), Digitale Arbeit, digitaler Wandel, digitaler Mensch?

10. Schlicher K, Paruzel A, Steinmann B, Maier GW (2018) Change Management für die Einführung digitaler Arbeitswelten. In: Maier GW, Engels G, Steffen E (Hrsg), Handbuch Gestaltung digitaler und vernetzter Arbeitswelten. Springer, Berlin. https://doi. org/10.1007/978-3-662-52903-4_16-1

11. Gausemeier J, Pfänder T, Thielemann F, Kespohl HD (2018) Innovationen für die Märkte von morgen: strategische Planung von Produkten, Dienstleistungen und Geschäftsmodellen. Carl Hanser Verlag, München

12. Venkatesh V, Bala H (2008) Technology acceptance model 3 and a research agenda on interventions. Dec Sci 39:273-315. https://doi.org/10.1111/j.1540-5915.2008.00192.x

13. Mlekus L, Bentler D, Paruzel A, Kato-Beiderwieden A - L, Maier GW (2020). How to raise technology acceptance: User experience characteristics as technology-inherent determinants. Gruppe. Interaktion. Organisation. Zeitschrift für Angewandte Organisationspsychologie (GIO). https://doi.org/10.1007/s11612-020-00529-7

14. Bentler D, Mlekus L, Paruzel A, Bansmann M, Foullois M, Jenderny S, Woeste L, Dumitrescu R, Röcker C, Maier GW (2019) Einführung von Augmented Reality in der Produktentstehung. Technische Realisierung und Change-Management als Erfolgsfaktor für den Veränderungsprozess. In: Gesellschaft für Arbeitswissenschaft e.V. (Hrsg) Arbeit interdisziplinär analysieren - bewerten - gestalten

15. Paruzel A, Bentler D, Schlicher K, Nettelstroth W, Maier GW (2020) Employee first, technology second: Implementation of smart glasses in a manufacturing company. Zeitschrift für Arbeits- und Organisationspsychologie 64(1):46-57. https://doi.org/10.1026/0932-4089/ a000292

16. Mlekus L, Maier GW (in prep) Not everyone benefits from technological advancements: Associations with competency requirements and employee reactions in two occupations

17. Koch A, Westhoff K (2012) Task-Analysis-Tools (TAToo) - Schritt für Schritt Unterstützung zur erfolgreichen Anforderungsanalyse. Pabst Science, Lengerich

18. Kato-Beiderwieden A-L, Schlicher KD, Ötting SK, Maier GW (in prep) Prospektive Kompetenzanalyse (ProKA) - Ein Verfahren zur Einschätzung von zukünftigen Kompetenzveränderungen

19. Jenderny S, Foullois M, Kato-Beiderwieden A, Bansmann M, Wöste L, Lamß J, Maier GW, Röcker C (2018) Development of an instrument for the assessment of scenarios of work 4.0 based on sociotechnical criteria. In: Proceedings of the 11th PErvasive Technologies Related to Assistive Environments Conference-PETRA, Corfu. ACM Press, New York, S 319-326

20. Mlekus L, Paruzel A, Bentler D, Jenderny S, Foullois M, Bansmann M, Woeste L, Röcker C, Maier GW (2018) Development of a change management instrument for the implementation of technologies. Technologies 6(4):120. https://doi.org/10.3390/technologies6040120

21. Bansmann M, Foullois M, Röltgen D, Woeste L, Dumitrescu R (2019) Reference architecture and classification of technology induced scenarios of digitized work. In: Proceedings of the 28th International Association for Management of Technology IAMOT, Mumbai, Indien 
Open Access Dieses Kapitel wird unter der Creative Commons Namensnennung 4.0 International Lizenz (http://creativecommons.org/licenses/by/4.0/deed.de) veröffentlicht, welche die Nutzung, Vervielfältigung, Bearbeitung, Verbreitung und Wiedergabe in jeglichem Medium und Format erlaubt, sofern Sie den/die ursprünglichen Autor(en) und die Quelle ordnungsgemäß nennen, einen Link zur Creative Commons Lizenz beifügen und angeben, ob Änderungen vorgenommen wurden.

Die in diesem Kapitel enthaltenen Bilder und sonstiges Drittmaterial unterliegen ebenfalls der genannten Creative Commons Lizenz, sofern sich aus der Abbildungslegende nichts anderes ergibt. Sofern das betreffende Material nicht unter der genannten Creative Commons Lizenz steht und die betreffende Handlung nicht nach gesetzlichen Vorschriften erlaubt ist, ist für die oben aufgeführten Weiterverwendungen des Materials die Einwilligung des jeweiligen Rechteinhabers einzuholen.

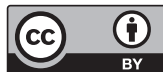

